\title{
The Effectiveness of D-branes in the Description of Near-Extremal Black Holes
}

\author{
Sumit R. Dast \\ Tata Institute of Fundamental Research \\ Homi Bhabha Road, Bombay 400 005, INDIA
}

\begin{abstract}
It is known that weak coupling calculations of absorption or emission by slightly non-extremal D-brane configurations are in exact agreement with semiclassical results for the black holes they describe at strong couplings. We investigate one open string loop corrections to processes involving single and parallel D-branes and show that a class of relevant terms vanish, indicating that these processes are not renormalized. Our results have implications for five dimensional black holes and extremal 3-branes.
\end{abstract}

March, 1997

1 E-mail: das@theory.tifr.res.in 


\section{Introduction}

Recently the idea that massive string states become black holes when the coupling is large [1] [2] [3] has been very successful. In particular, five dimensional extremal black holes with large horizons are described by bound states of D-branes whose degeneracies exactly reproduce the Beckenstein-Hawking entropy [4]. This result was extended to four dimensional extremal black holes [5] and to spinning black holes [6].

The entropy and hawking temperature continue to agree in the near extremal limit [7], [8]. It was also found that the lowest order decay rate of slightly nonextremal Dbrane configurations is proportional to the horizon area [7] which is consistent with the semiclassical Hawking radiation from such holes, as shown in [9]. Rather surprisingly the two emission (and absorption) rates for neutral scalars were in fact found to agree exactly [10]. Exact agreements were also found for neutral and charged scalars in five and four dimensional black holes [11]. Even more remarkably, the grey body factors which describe a nontrivial energy dependence of the absorption cross-section at higher energies are also in exact agreement [12], a result verified in the four dimensional case as well [13]. A general analysis of classical absorption by such black holes and the possibility of agreement of classical and D-brane greybody factors, has been carried out in [14].

A more detailed test of these ideas is provided by the emission and absorption of "fixed" scalars by five dimensional black holes [15], where agreement between the D-brane and classical calculations were demonstrated in [16]. The grey body factors at higher energies agree as well [17].

A related example where there seem to be exact agreement of D-brane and general relativity results is the absorption of $l=0$ and $l=1$ waves by extremal 3 -branes with no momentum [18] [19]. The cross-sections for higher partial waves agree upto numerical factors [19]. In this case the near-extremal entropy also differs by a numerical factor [20].

For systems arbitrarily far from extremality (like the Schwarzschild black hole) it has been argued that the microscopic and semiclassical answers should match only at a special value of the coupling at which the horizon curvature is of the string scale [1], [21]. It was shown in 21] that in all known cases the stringy and semiclassical entropies indeed match at this point, upto numerical factors.

The reason why these results appear surprising is that the D-brane calculations are performed in the lowest order of the open string perturbation theory, using in fact a low energy effective action, while the brane configurations describe large black holes only when 
the open string coupling is large. For extremal BPS holes, nonrenormalization theorems allow an extrapolation of weak coupling results to strong coupling. However away from extremality there does not appear to be an obvious reason why this can be done.

Consider for example the five dimensional near extremal black hole which is described by bound states of 1D-branes and 5D-branes with some momentum flowing along the $1 \mathrm{D}$ brane. Non extremality is introduced by allowing both left and right momenta on the 1D brane and the classical solution of the low energy effective action has a ten-dimensional string metric

$$
\begin{aligned}
d s^{2} & =\left(1+\frac{r_{1}^{2}}{r^{2}}\right)^{-1 / 2}\left(1+\frac{r_{5}^{2}}{r^{2}}\right)^{-1 / 2}\left[d t^{2}-d x_{5}^{2}-\frac{r_{0}^{2}}{r^{2}}\left(\cosh \sigma d t+\sinh \sigma d x_{5}\right)^{2}\right] \\
& -\left(1+\frac{r_{1}^{2}}{r^{2}}\right)^{1 / 2}\left(1+\frac{r_{5}^{2}}{r^{2}}\right)^{-1 / 2}\left[d x_{1}^{2}+d x_{2}^{2}+d x_{3}^{2}+d x_{4}^{2}\right] \\
& -\left(1+\frac{r_{1}^{2}}{r^{2}}\right)^{1 / 2}\left(1+\frac{r_{5}^{2}}{r^{2}}\right)^{1 / 2}\left[\left(1-\frac{r_{0}^{2}}{r^{2}}\right)^{-1} d r^{2}+r^{2} d \Omega_{3}^{2}\right]
\end{aligned}
$$

The various length scales are given in terms of the charges by

$$
\begin{array}{lr}
r_{1}^{2}=\frac{16 \pi^{4} \alpha^{\prime 3}\left(g Q_{1}\right)}{V} & r_{5}^{2}=\alpha^{\prime}\left(g Q_{5}\right) \\
\frac{1}{2} r_{0}^{2} \sinh 2 \sigma=\frac{16 \pi^{4} \alpha^{\prime 4}\left(g^{2} N\right)}{R^{2} V} & r_{N}^{2}=r_{0}^{2} \sinh ^{2} \sigma
\end{array}
$$

Here $\alpha^{\prime}=1 /(2 \pi T)$ where $T$ is the elementary string tension, $g$ is the string coupling. The brane configuration lies on a $T^{4} \times S^{1}$ with the one brane along the $S^{1}$. The radius of this $S^{1}$ is $R$, while the volume of the $T^{4}$ is $V$. The integers $Q_{1}, Q_{5}, N$ are the 1 -brane $\mathrm{RR}$ charge, 5-brane RR charge and the total momentum. The extremal limit is $r_{0} \rightarrow 0$ and $\sigma \rightarrow \infty$ with $r_{N}$ held fixed.

It is clear from the classical solution that the classical limit of the string theory corresponds to $g \rightarrow 0$ with $g Q_{1}, g Q_{5}, g^{2} N$ held fixed [12]. In fact we have large black holes (compared to string scale) when $g Q_{1}, g Q_{5}, g^{2} N>1$ and small holes when $g Q_{1}, g Q_{5}, g^{2} N<$ 1. It is in the latter regime that the D-brane description is good.

Another example which we will consider in the following is the self-dual 3-brane in Type IIB theory. The extremal solution has a zero horizon area, but is completely nonsingular and the dilaton is a constant in the classical solution. The extremal string metric is given by

$$
d s^{2}=A^{-1 / 2}\left(-d t^{2}+d x_{1}^{2}+d x_{2}^{2}+d x_{3}^{2}\right)+A^{1 / 2}\left(d r^{2}+r^{2} d \Omega_{5}^{2}\right)
$$


with

$$
A(r)=1+\frac{4 \pi \alpha^{\prime 2}(g N)}{r^{4}}
$$

where $N$ is the RR charge. The curvature at the horizon $r=0$ is $\sim 1 /\left[\alpha^{\prime} \sqrt{g N}\right]$. Thus when $g N$ is large, the curvatures are small and one may trust the supergravity limit. Note that the parameters in the classical solution depends on $g$ and $\alpha^{\prime}$ only through the combination $\kappa \sim g \alpha^{\prime 2}$ where the ten dimensional Newton constant is $G_{10}=8 \pi \kappa^{2}$. For parallel branes of one type this happens only for 3-branes.

To appreciate the meaning of these products let us consider a simpler example in scalar $\phi^{3}$ field theory where we are interested in describing classical solutions in the presence of a static source 2

$$
\nabla^{2} \phi+\lambda \phi^{2}=\kappa \delta(\vec{x})
$$

By scaling the field $\phi$ it is easily seen that the solution is of the form

$$
\phi(x)=\frac{1}{\lambda} F(x ; \lambda \kappa)
$$

so that the solution is characterized by the product $\lambda \kappa$. The classical limit is $\lambda \rightarrow 0$ and a solution which captures the nonlinearity is characterized by $\lambda \kappa=$ fixed. One may obtain the classical solution by summing over all tree level Feynman diagrams with arbitrary number of insertions of the source.

The product $\lambda \kappa$ is in fact an exact analog of the products $g Q$ in the D-brane context in the dilute gas regime $r_{N}<<r_{1}, r_{5}$, as clear from the classical solution. The full classical solution can be obtained by summing over an infinite number of string diagrams which does not contain any closed string loop, but contains all terms with closed strings terminating on an aribtrary number of branes. Each such insertion carries a factor $g Q$ which has to be held finite. In other words we have to sum over all open string loops. Closed string loops do not contain any factor of the charge $Q$ and are therefore suppressed. This perturbation expansion is a description of the black hole expanded around flat space-time with the curvature emerging as a result of summing over open string loops.

It is important to understand why the tree level results are in exact agreement with answers which are expected to hold for strong couplings. The point is that even though the near extremal states break supersymmetry, for slight non-extremality these are states in a supersymmetric background. In such situations it is natural to expect that there

2 This analogy was suggested by A. Jevicki. 
are nonrenormalization theorems which protect some tree level results from acquiring loop corrections. Some of these issues can be addressed in the worldbrane low energy effective theory. Indeed such considerations have been used by Maldacena [22] to argue that processes which follow from the moduli space approximation are not renormalized to any order. However, these arguments cannot be used to justify the success of the calculation of other cross-sections like the absorption or emission of fixed scalars in the five dimensional black holes and higher partial waves in the 3-brane.

In the following we explicitly examine one open string loop corrections to some absorption and emission processes from single and parallel branes. The restriction to parallel branes comes from the fact that it is for these that we can perform string theoretic calculations explicitly. Our results are directly relevant to 3-branes. Moreover since the 1-brane 5-brane system which describes the five dimensional black hole reduces to a single long D-string, our results have some relevance to the five dimensional black hole as well. It must be remembered, though, that the use of an effective string at the loop level needs further justification.

\section{The D-brane calculation}

The D-brane calculation of emission from slightly non-extremal configurations is performed in the lowest order of the open string perturbation theory and at low energies. Let us identify precisely the approximations used here.

As shown in 23] the 1-brane 5-brane system with large momentum along the string direction must be regarded as multiply wound along the lines of [24], 25] so that we essentially have a single long string which is wrapped around a large circle of radius $Q_{1} Q_{5} R$. In the following we will therefore model this system by such a D-string. The string tension

of this effective string should be then fractional $T_{\text {eff }}=1 /\left(2 \pi \alpha^{\prime} Q_{5}\right)$ [23], [26]. Furthermore, the massless modes are now open string modes whose polarizations are restricted to lie along the $T^{4}$ directions orthogonal to the string direction $S^{1}$, together with their fermionic partners.

The D-brane calculations are in fact performed in this effective D-string theory, using the Dirac-Born-Infeld (DBI) effective action

$$
S=\frac{T_{e f f}}{2} \int d^{2} \sigma e^{-\phi} \sqrt{\operatorname{det}\left(G_{\mu \nu}^{S} \partial_{\alpha} X^{\mu} \partial^{\alpha} X^{\nu}\right)}
$$


where $G_{\mu \nu}^{S}$ is the bulk string frame metric and $\phi$ is the ten dimensional dilaton. Choosing the static gauge $\tau=X^{0} \equiv x^{0}, \sigma=X^{5} \equiv x^{5}$ and keeping only the components $X^{I}$ where $I=1, \cdots 4$ are the four $T^{4}$ directions and retaining terms upto four derivatives on the open string fields fields $X^{I}$ the DBI action becomes

$$
\begin{aligned}
S=\frac{T_{e f f}}{2} \int d X^{0} d X^{5} e^{-\phi}[1+ & \frac{1}{2} G_{I J} \partial_{+} X^{I} \partial_{-} X^{J} \\
& \left.-\frac{1}{8} G_{I J} G_{K L}\left(\partial_{+} X^{I} \partial_{+} X^{J}\right)\left(\partial_{-} X^{K} \partial_{-} X^{L}\right)\right]
\end{aligned}
$$

where $x^{ \pm}=x^{0} \pm x^{5}$. The effective action (2.2) can be derived by considering open string amplitudes on a disc, as verified explicity for the second term of (2.2) in [27].

Note that the metric $G_{I J}$ is generally a function of all the coordinates

$$
G_{I J}\left[x^{0}, x^{5}, X^{I}\left(x^{0}, x^{5}\right), X^{m}\left(x^{0}, x^{5}\right)\right]
$$

(where $m=6 \cdots 9$ ), so that even the term $\partial X^{I} \partial X^{J}$ contains an arbitrary number of open string fields. For emission of S-wave modes, the low energy approximation amounts to ignoring the dependence of the bulk metric on $X^{I}$ and $X^{m}$. (The dependence on $x^{0}$ and $x^{5}$ of course remain which imposes momentum conservation in the Neumann directions). This amounts to retaining terms which contain the least number of momenta with a given number of open string fields. We will therefore expand

$$
G_{I J}=\delta_{I J}+\sqrt{2} \kappa h_{I J}\left(x^{0}, x^{5}\right)
$$

The BPS states of the system correspond to a background value of only the left moving part of the $X^{I}$ 's. Non-BPS states correspond to addition of pairs of oppositely moving modes [24]. In the dilute gas region, and in the lowest order of the open string coupling, one has a standard thermodynamics problem of a one (spatial) dimensional gas of bosons and fermions with some given energy and total momentum. The various thermodynamic quantities like the entropy and the temperature may be calculated in this gas using standard methods [10] and, as is well known by now, for near-BPS situations the results are in perfect agreement with the semiclassical answers obtained from the metric (1.1) [7], [8].

For the system of $N$ parallel 3-branes, the extreme low energy effective worldvolume action is a four dimensional $N=4 U(N)$ Yang-Mills theory [28] with background fields [29]. Emission of higher angular momentum scalars may be studied by retaining the dependence of the background fields on the transverse coordinates, as we will see soon. The situation studied so far is the extremal state with no momentum. 


\subsection{Minimally coupled scalars for the 1-5 brane}

First consider the emission of minimally coupled scalars. Examples of these are transverse traceless components of $h_{I J}$. In the bulk theory these modes obey the minimally coupled Klein-Gordon equation in the five dimensional geometry. For this emission we can set the dilaton field to zero and in fact concentrate on one particular component of $h_{I J}$. The relevant interaction term which involves the miminum number of derivatives of open string field comes from the second term in $(2.2)$

$$
L_{i n t}^{(1)}=\sqrt{2} \kappa h_{I J} \partial_{+} X^{I} \partial_{-} X^{J}
$$

and describes the annahilation of two oppositely moving open strings into a closed string state described by $h_{I J}$. At low energies, $h_{I J}$ is considered as a function of only the longitudinal coordinates $x^{0}, x^{5}$.

When the outgoing particle does not have any momentum in the $X^{5}$ direction, it is a neutral scalar from the five dimensional point of view. The tree level emission rate into a closed string state of momenta $k$ (note all the $k^{i}$ with $i=1 \cdots 5$ are zero), when averaged over the initial states is [10] (the notation is of [12])

$$
\Gamma(k)=2 \pi^{2} r_{1}^{2} r_{5}^{2} \frac{\pi k_{0}}{2} \frac{1}{\left(e^{\frac{k_{0}}{2 T_{L}}}-1\right)\left(e^{\frac{k_{0}}{2 T_{R}}}-1\right)} \frac{d^{4} k}{(2 \pi)^{4}}
$$

$T_{L}$ and $T_{R}$ are the temperatures of the left and right moving modes on the effective string. The temperature, which is the same as the Hawking temperature is then given by

$$
\frac{1}{T}=\frac{1}{2}\left(\frac{1}{T_{L}}+\frac{1}{T_{R}}\right)
$$

Detailed balance then gives an absorption cross-section

$$
\sigma\left(k_{0}\right)=2 \pi^{2} r_{1}^{2} r_{5}^{2} \frac{\pi k_{0}}{2} \frac{\left(e^{\frac{k_{0}}{T}}-1\right)}{\left(e^{\frac{k_{0}}{2 T_{L}}}-1\right)\left(e^{\frac{k_{0}}{2 T_{R}}}-1\right)}
$$

When we restrict to extremely low energies $k_{0}<<T_{L}, T_{R}$ (but $k_{0} \sim T$ ) one has $\sigma=$ $A_{H}$, the area of the horizon, and this is exactly what we get from a classical absorption calculation for any spherically symmetric black hole in any number of dimensions [30].

In fact, the entire expression (2.8) (with $k_{0} \sim T_{L}, T_{R}$ ) agrees exactly with the classical grey body factor calculated in the dilute gas regime

$$
r_{0}, r_{N}<<r_{1}, r_{5}
$$


and $k_{0} \omega_{5}<<1$, i.e. at wavelengths much larger than the gravitational radius [12]. In fact, beyond the dilute gas approximation detailed agreements of D-brane and classical grey body factors are not always present [31] [32]. Even in the dilute gas regime there is some disagreement for charged particle emission when the individual energies and charges are large, but the difference is small [32].

One important feature of this calculation must be emphasized. Since the interaction term (2.5) involves only two open string fields and the effective tension $T_{\text {eff }}$ sits outside the action, the amplitude is independent of $T_{\text {eff }}$. The decay rate does depend on the length of the effective string and the agreement with the semiclassical answer holds when $L_{e f f}=2 \pi Q_{1} Q_{5} R$.

\subsection{Fixed scalars in the 1-brane 5-brane}

A more detailed test is provided by looking at fixed scalars which do not obey the minimally coupled Klein-Gordon equation. An example of this is the size of the $T^{4}$. To analyze this one has to substitute

$$
G_{I J}^{S}=e^{2 \nu} \delta_{I J}
$$

in equation (2.2). Since this is the trace part of the ten dimensional graviton, it mixes with the dilaton. The requirement that no five dimensional dilaton is emitted means that one has to set $\phi=2 \nu$ [16]. It is clear from (2.2) that $\nu$ couples only to the quartic term in the action. The DBI action becomes, upto terms linear in $\nu$,

$$
S=\frac{T_{e f f}}{2} \int d x^{0} d x^{5}\left[1+\frac{1}{2} \partial_{+} X^{I} \partial_{-} X_{I}-\frac{1}{8}\left(\partial_{+} X\right)^{2}\left(\partial_{-} X\right)^{2}-\frac{1}{4} \nu\left(\partial_{+} X\right)^{2}\left(\partial_{-} X\right)^{2}\right]
$$

Thus the emission of a quanta of $\nu$ has the important characteristic that the lowest order process involves four open string fields. For the same reason the effective string tension $T_{\text {eff }}$ does not scale out and the answer depends on $T_{\text {eff }}$.

Once again, the field $\nu$ has to be treated as a function of $x^{0}$ and $x^{5}$ only. It was shown in [16] that the emission rate is in exact agreement with the semiclassical answer provided $T_{\text {eff }}=1 /\left(2 \pi \alpha^{\prime} Q_{5}\right)$ and $L_{e f f}=2 \pi Q_{1} Q_{5} R$ as expected from earlier considerations. 


\subsection{Absorption by 3-branes}

In the above examples, there is as yet no complete derivation of the effective string model from the microscopic model of branes. From this point of view it is useful to consider parallel branes of a single type which lead to nonsingular geometries. An example is the self dual 3-brane in Type IIB theory. Consider for example the absorption of dilatons by an extremal 3-brane. The relevant interaction comes from a term involving two worldvolume gauge fields going into a dilaton [18]

$$
S_{\text {int }} \sim-\frac{\kappa}{\sqrt{2}} \int d^{4} x \phi\left(x^{\alpha}, X^{i}\right) \operatorname{Tr}\left[\partial_{\alpha} A_{\beta} \partial^{\alpha} A^{\beta}\right]
$$

where $x^{\alpha}$ are the four brane coordinates and $X^{i}$ denote the transverse coordinates. $A_{\alpha}$ is the gauge field.

The emission of dilatons in some given partial wave $l$ is described by the following term in the Taylor expansion of $\phi\left(x^{\alpha}, X^{i}\right)$ in powers of $X^{i}$ [18, 19]

$$
L_{l} \sim\left(\partial_{i_{1}} \cdots \partial_{i_{l}} \phi\right)_{0} \operatorname{Tr}\left[<X^{i_{1}} \cdots X^{i_{l}}>F_{\alpha \beta}^{2}\right]
$$

where $<X^{i_{1}} \cdots X^{i_{l}}>$ denotes the projection of the products to the definite angular momenta, and the derivatives on $\phi$ are evaluated at the location of the brane at $X^{i}=0$. Similar considerations apply to other closed string states like the longitudinally polarized graviton and the RR scalar [19].

It is clear from the classical solution that the expansion parameter in the classical absorption cross-section of a wave of frequency $\omega$ is the combination $\omega\left(\alpha^{\prime 2} g N\right)^{1 / 4}$. It has been shown in [19] that for the $l$ th partial wave the answer is

$$
\sigma^{l}(\omega) \sim \omega^{(4 l+3)}\left(\alpha^{\prime 2} g N\right)^{l+2}
$$

Remarkably, for $l=0,1$ the cross-section exactly matches the D-brane answer, whereas for $l=2,3$ the two answers differ by a numerical factor [19].

\section{Strategy for higher loops}

In the following we will examine lowest order open string corrections to absorption/emission processes from slightly non-extremal single branes and parallel branes. In these situations explicit string theory calculations can be performed and these are directly 
relevant to the absorption by three branes. Moreover the 1-brane 5-brane system reduces to a single long D-string at low energies. Consequently some of our results will be relevant for this case as well.

To appreciate the issue of loop corrections, consider for simplicity black holes which contain a single length scale in the problem. Examples are extremal branes (the 3-brane in particular) and the five dimensional black hole in the special case $r_{1}=r_{5}=r_{N}=R$. Let us call this length scale $l$. In the classical solution the string coupling can enter only through this length scale $l$ which is typically given by the form

$$
l^{(d-3)} \sim g Q \alpha^{(d-3 / 2)}
$$

where $d$ denotes the number of non-compact dimensions. It is then clear that the classical absorption cross-section has to be of the form

$$
\sigma_{\text {class }} \sim l^{d-2} F\left(\omega^{(d-3)} g Q \alpha^{(d-3 / 2)}\right)
$$

On general grounds we expect that this classical answer should agree with the D-brane answer when $g Q$ is large. However the above expression shows that for sufficiently small $\omega$ one may have the factor $\omega^{(d-3)} g Q \alpha^{\prime(d-3 / 2)}$ small even if $g Q$ is large so that one may imagine performing a Taylor expansion of the function $F$, which then becomes a power series expansion in the string coupling $g$ as well [18]. The spectacular success of the tree level D-brane calculations of the absorption cross-section then means that the lowest order term in this expansion has been shown to agree with the lowest order term in D-brane open string perturbation theory.

The puzzle regarding this agreement of D-brane and classical calculations may be now restated as follows : In the classical limit a higher power of the string coupling comes with a higher power of the energy in a specific way dictated by (3.2). On the other hand, on the D-brane side these higher powers of coupling are to be obtained in open string perturbation theory and there is no a priori reason why this should also involve higher powers of energy in precisely the same way.

This implies that the following two kinds of loop corrections must be absent for the correspondence to work. (1) For a given absorption or emission process open string loop diagrams with the same external states must be suppressed at low energies compared to the tree diagram. (2) Suppose we concentrate on emission of some given closed string state and let the leading order tree process give a cross-section $\sigma \sim g^{\alpha}$ with some energy 
dependence. For $\alpha$ large enough it is possible that there is a string loop process with the same dependence on the coupling 3. This must, of course, involve external states which are different from the tree process. For processes where the D-brane tree level calculation gave the correct answer such loop corrections must be suppressed at low energies.

What we want to calculate is the (open) string loop correction to the effective action of the massless open string modes in the presence of a background closed string which we will take to be the ten dimensional metric or the dilaton. As we will see soon these diagrams are generically nonzero. However it must be remembered that we have compared processes only at low energies. We have to see whether there are loop corrections at such low energies.

For S-wave emission/absorption low energies meant the minimal powers of momenta of the open string states and no powers of momenta for the closed string states. In oher words when the effective action is expressed in the presence of closed string fields on the brane there were no derivatives with respect to either the longitudinal or transverse directions. To examine whether these specific processes receive a one-loop correction it is sufficient to consider the effective action in flat space. Then the curved space effective action may be obtained by simply replacing the flat metric by the curved metric. This follows from general coordinate invariance - in fact the equivalence principle. For example for the case of a D-string, if we know the term in the flat space effective action

$$
\partial_{+} X^{I} \partial_{-} X^{J} \delta_{I J}
$$

Then in the curved space effective action, $\delta_{I J}$ may be replaced by any tensor made out of the metric, e.g.

$$
\partial_{+} X^{I} \partial_{-} X^{J}\left[G_{I J}+R_{I J}+\cdots\right]
$$

where $R_{I J}$ is the Ricci tensor. However only the first term in (3.4) does not involve any derivative of the metric. Thus at low energies only the first term is relevant, which may be obtained by simply replacing the flat metric $\delta_{I J}$ by the curved metric $G_{I J}$. In a similar fashion once we figure out the term in the flat space effective action

$$
\partial_{+} X^{I} \partial_{+} X^{J} \partial_{-} X^{K} \partial_{-} X^{L} \delta_{I J} \delta_{K L}
$$

3 The importance of this was emphasized to me by I. Klebanov. 
we will know the loop corrections to the term which was responsible for emission of fixed scalars. The fact that it is sufficient to consider the effective action in flat space implies that there is enhanced supersymmetry in the problem 6 .

We are interested in low energies of the open string modes as well. This means that in a term with some number of open string fields we keep the term with the minimal number of derivatives on these fields. This means we are not interested in terms like

$$
\partial^{2} X^{I} \partial^{2} X^{J} \quad \partial^{2} X^{I} \partial^{2} X^{J} \partial_{+} X^{K} \partial_{-} X^{L} \cdots
$$

and so on.

To find whether higher loop processes involving different external states may correct a tree level emission/absorption of some given closed string state, we have to include insertions of closed string backgrounds. Nevertheless the calculation will be simplified by requiring that we look at the term which has the required powers of the momentum.

Loop diagrams with insertions of closed string states are also essential to study possible corrections to the absorption/emission in higher partial waves. This is because for such processes the tree level term itself involves derivatives on the closed string fields (see e.g. $(2.13))$.

There are, however, other S-wave processes where the low energy curved space effective action cannot be read off from the flat space action. These involve situations where the worldsheet is curved. Recall that we are using the static gauge, so that certain components of the background metric become worldsheet metrics. In the five dimensional black hole an example is the component $h_{55}$. It follows from the Born-Infeld action that this has couplings of the form

$$
h_{55}\left[\left(\partial_{+} X\right)^{I}\left(\partial_{+} X_{I}\right)+\left(\partial_{-} X\right)^{I}\left(\partial_{-} X_{I}\right)\right]
$$

i.e. this couples to a sum of chiral operators. Such operators cannot appear in the DBI action in flat backgrounds. For such fields the loop corrections cannot be obtained from the flat space terms.

\footnotetext{
4 This was pointed out by S. Shenker.
} 


\section{The One Loop Calculation : Flat space}

We will first analyze open string loop diagrams without any closed string insertion. We will use the light cone gauge Green-Schwarz formalism. To do this, however, we have to consider the brane directions to be euclidean and one of the transverse directions to be time. Such a framework has been discussed in [33]. For a $p$-brane we thus have $\left(X^{1} \cdots X^{p+1}\right)$ to be the longitudinal directions, $\left(X^{p+2} \cdots X^{8}, X^{ \pm}=X^{0} \pm X^{9}\right)$ as the transverse directions. This specifies the boundary conditions for the bosonic fields of the open string in the standard fashion. The boundary conditions for the fermions are determined by the requirement that half of the supersymmetries are unbroken and are given by

$$
S_{+}^{a}(\sigma, \tau)=M_{b}^{a} S_{-}^{b}(\sigma \cdot \tau) \quad \sigma=0, \pi
$$

The matrix $M$ may be written in terms of the ten dimensional gamma matrices [33], [34], [35]. For our purposes we will need the properties

$$
\begin{aligned}
& M^{T} \gamma^{I} M=-\gamma^{I} \quad(I=(p+2) \cdots 8) \\
& M^{T} \gamma^{\alpha} M=\gamma^{\alpha} \quad(\alpha=1 \cdots p) \\
& M^{T} M=I
\end{aligned}
$$

The vertex operators for the open string massless states with polarizations in the Dirichlet and Neumann directions are given by (respectively)

$$
\begin{aligned}
& V_{D}=\zeta_{I}(k)\left(\partial_{\sigma} X^{I}-S_{+} \gamma^{I \alpha} S_{+} k_{\alpha}\right) e^{i k X} \\
& V_{N}=\zeta_{\beta}(k)\left(\partial_{\tau} X^{\beta}-S_{+} \gamma^{\beta \alpha} S_{+} k_{\alpha}\right) e^{i k X}
\end{aligned}
$$

where we have used the fact that the momenta of the open string states are always in the Neumann direction.

\subsection{The quadratic term}

Let us first consider the terms in the one (open string) loop contribution to the effective action which involve two open string fields. When both the open string operators are on the same boundary we have a term like

$$
K_{(2,0)} \operatorname{Tr}\left[V\left(k_{1}\right) \Delta V\left(-k_{1}\right) \Delta\right]
$$


where $\Delta$ denotes the open string propagator and $V$ stands for either $V_{D}$ or $V_{N}$. Here $K_{(2,0)}$ is the Chan-Paton factor. For a single brane this is just the $U(1)$ charge, whereas for multiple branes we have the usual trace over the $U(N)$ group generators.

Since we are dealing with an oriented string theory one has to add the contribution from the case where they attach to the two different boundaries, which is obtained by insertion of two twist operators $\Omega$

$$
K_{(1,1)} \operatorname{Tr}\left[V\left(k_{1}\right) \Omega \Delta V\left(-k_{1}\right) \Omega \Delta\right]
$$

The Chan Paton factor $K_{(1,1)}$ is nonzero only for the overall $U(1)$.

It is well known that the terms (4.4) and (4.5) individually vanish. This happens because the trace involves a trace over the zero modes of the fermion fields $S_{0}^{a}$. Only products of at least eight zero modes have a nonzero trace, while the terms like (4.4) can involve only four such zero modes. This is in fact a reflection of supersymmetry of the underlying theory.

Thus, to lowest order in the energy there are no one loop correction to the effective action involving two open string fields. This result is valid for parallel branes of any type. In particular, this establishes that the lowest order S-wave dilaton absorption by 3-branes is not renormalized to this order. In so far as the 1-brane 5-brane system can be regarded as a single long D-string, this also implies that the emission/absorption of minimal scalars in the five dimensional black hole is not renormalized.

This result is an explicit verfication of the non-renormalization arguments in [22].

\subsection{The quartic term}

Now consider terms in the effective action which involve four open string fields - again in flat space. The planar annulus amplitude is given by

$$
K_{(4,0)} \operatorname{Tr}\left[V\left(k_{1}\right) \Delta V\left(k_{2}\right) \Delta V\left(k_{3}\right) \Delta V\left(k_{4}\right) \Delta\right]
$$

where $K_{(4,0)}$ is the relevant Chan-Paton factor. Since each vertex operator involves two

fermion zero modes, (4.6) has a single term with eight fermion zero modes so that the vertex operators appearing in (4.6) may be replaced by

$$
V \sim S_{0} \gamma^{\mu \alpha} S_{0} k_{\alpha} \zeta_{\mu}(k) e^{i k X}
$$


We are looking for a term which has four powers of open string momenta - one for each open string field. This means that we can ignore the $e^{i k X}$ factor in (4.7) and use

$$
V \sim S_{0} \gamma^{\mu \alpha} S_{0} k_{\alpha} \zeta_{\mu}(k)
$$

The final nonzero result can be easily computed following the procedures in for example [36]. Let us call this $\mathcal{A}_{2}$.

As before, we have to add the contributions from the non-planar diagrams which may be obtained by putting in an even number of twist operators in (4.6). For example a nonplanar diagram with three vertices on one of the boundaries and one vertex on the other boundary is given by

$$
K_{(3,1)} \operatorname{Tr}\left[V\left(k_{1}\right) \Omega \Delta V\left(k_{2}\right) \Omega \Delta V\left(k_{3}\right) \Delta V\left(k_{4}\right) \Delta\right]
$$

where $K_{(3,1)}$ is the relevant Chan-Paton factor in this case. Using $\Omega^{2}=1$ the effect of a twist operator is easily seen to result in a change of sign of of the odd oscillators, $\alpha_{n} \rightarrow(-1)^{n} \alpha_{n}, S_{n} \rightarrow(-1)^{n} S_{n}$ in all the vertex operators on one of the boundaries. For (4.9) this means that the odd oscillators in $V\left(k_{2}\right)$ have to be flipped.

However, as we have just found, at low open string energies there are no oscillators in the vertex operators! Thus, at low energies, the individual contributions of each of the nonplanar diagrams is the same as that of the planar diagrams apart from (i) combinatoric factors and (ii) Chan-Paton factors. There are 2 terms with all the vertices on the same boundaries, 8 terms with three vertices on one boundary and one vertex on the other boundary and 6 terms with two vertex operators on each boundary. Thus we get the net contribution

$$
\left(2 K_{(4,0)}+8 K_{(3,1)}+6 K_{(2,2)}\right) \mathcal{A}_{2}
$$

This is in general nonzero.

However, when we have a single brane, the gauge group is $U(1)$ and the two ends of the open string going around the loop have equal and opposite charges $\epsilon$. In this case

$$
K_{(4,0)}=-K_{(3,1)}=K_{(2,2)}=\epsilon^{4}
$$

and the net contribution (4.10) vanishes.

We emphasize that this result is true for the lowest order term in the open string energy. At higher energies the $e^{i k X}$ factors in $V$ cannot be set to 1 and the vertex operators 
will contain oscillators. As a result the magnitude of a non-planar diagram will be different from a planar diagram and the above cancellation will not take place.

Nonrenormalization properties of $F^{4}$ terms have been shown for the Type I superstring and follow from cancellations between the annulus and the mobius strip diagrams [37]. Similar nonrenormalization theorems are also required for the consistency of M(atrix) theory [38]. However, as we discuss below, the terms which are required to be protected in this case are of a rather different nature than the terms we have been discussing.

\subsection{Relationship to brane dynamics}

For single branes, the one loop calculation in the previous section is closely related to the dynamics of two branes which are moving and have waves on it as well. In this situation, the force between these branes may be calculated by evaluating an annulus diagram in the open string theory where the two boundaries of the worldsheet are attached to the two different branes. The worldsheet theory of the open strings connecting these branes now contain the terms

$$
e_{1} \int_{\sigma=0} d \tau A_{I}\left(X^{\alpha}\right)\left(\partial_{\sigma} X^{I}+\cdots\right)-e_{2} \int_{\sigma=\pi} d \tau A_{I}\left(X^{\alpha}\right)\left(\partial_{\sigma} X^{I}+\cdots\right)
$$

where $\alpha$ denotes a Neumann direction and $I$ denotes a Dirichlet direction and the dots represent fermionic terms. It is then easily seen that the annulus amplitudes we evaluated in the previous section are obtained by expanding the partition function in the presence of the additional terms (4.12) in powers of the backgrounds $A_{I}\left(x^{\alpha}\right)$ and (i) setting $e_{1}=e_{2}$ and (ii) setting the distance between the branes to zero.

Since we were interested in the situation where $\partial_{\alpha} A_{I}$ are constant, the $A_{I}$ 's we need to consider are those which are at most linear in the $X^{\alpha}$ 's.

When the background field is only a function of the time coordinate, i.e. $A_{I}\left(x^{0}\right)$ we have the two branes moving with velocities $e_{1}$ and $e_{2}$, a situation analyzed in [39]. In this case the zero result we found to order $A_{I}^{4}$ is simply a special case of the general result that the force between BPS branes vanish when the relative velocity is zero - which follows from Lorentz invariance and the fact that static BPS branes have no forces between them regardless of the distance of separation.

Our result is, however, more general than this situation. For a general $p$-brane we can transform our problem to the case of uniformly moving branes in only some special 
situations. Consider for example the case of $p=1$. In that case the general form of the background is

$$
A_{I}\left(x^{0}, x^{1}\right)=E_{I} x^{0}+B_{I} x^{1}
$$

When $E_{I}$ and $B_{I}$ are parallel to each other so that $E_{I}=E \hat{e}_{I}$ and $B_{I}=B \hat{e}_{I}$ the term in the action is simply

$$
\left(E X^{0}+B X^{1}\right) \partial_{\sigma}\left(\hat{e}_{I} X^{I}\right)
$$

Now we can perform a Lorentz transformation in the coordinates (provided $E^{2}>B^{2}$ ) to go to a new time variable $Y^{0}$ in terms of which we simply have

$$
\left(\sqrt{E^{2}-B^{2}}\right) Y^{0} \partial_{\sigma}\left(\hat{e}_{I} X^{I}\right)
$$

This is exactly the case which may be mapped to uniformly moving branes.

When $E_{I}$ and $B_{I}$ are not parallel this cannot be done any more and we have a situation which is physically quite different. There are no obvious reasons why the one loop corrections to the effective action vanish. It is, however, tempting to speculate that terms in the effective action with higher powers of the open string fields also do not receive any loop corrections. This would be relevant to the emission of particles with angular momentum 40].

The above discussion shows that the nonrenormalization property we are looking for is of a different nature than that required in $\mathrm{M}$ (atrix) theory. In the latter context one is interested in the case where two branes are moving with respect to one another. There is no tree level term and the entire contrbution is expetced to arise from one loop. In our case there is a tree level term which needs to be protected from loop corrections.

It may appear that our vanishing result for the abelian situation is simply a reflection of the fact that there are no charged fields in the theory. However we are looking at higher energy processes where the nonzero dipole moment of the open string might have led to nontrivial effects. In fact, by T-duality the problem may be related to the problem of open strings in constant electromagnetic backgrounds $\mathrm{O}$. In this T-dual situation it is well known that there is a one loop correction to the neutral bosonic string partition function in the presence of abelian constant fields. In fact one has 41]

$$
Z_{1-\text { loop }}(F)=\operatorname{det}[1+F] Z_{1-\text { loop }}(0)
$$

5 In doing this it must be remembered that the open string momenta continue to be only in the Neumann directions. 
$Z_{1-\text { loop }}(0)$, the zero field partition function is nonzero (in fact infinite due to the tachyon) for the bosonic string. For the superstring a similar formula is likely to hold 6 . Then $Z_{1-\text { loop }}(F)$ would vanish because $Z_{1-\text { loop }}(0)$ vanishes as a result of supersymmetry. This seems to suggest that in our problem the higher order terms in the open string fields would also vanish.

\section{Loop effects with closed string insertions}

We now consider the effect of closed string insertions on loops which could give contributions to the absorption processes discussed above. Potentially a one loop diagram with lower number of open string fields can contribute, in the same order of the string coupling $g$, to a process where the tree level involves a higher number of open strings.

It is clear that there cannot be any such correction to the emission of S-wave minimally coupled scalars in the five dimensional black hole. The tree level process for this is of order $g$ while the loop level process starts at order $g^{2}$

Consider for example the annulus diagram with two open strings and one closed string. The vertex operator for a massless bosonic closed string state is given by

$$
\left(\partial_{+} X^{\mu}-S_{+} \gamma^{\mu \alpha} S_{+} k_{\alpha}\right)\left(\partial_{-} X^{\nu}-\eta S_{+} \gamma^{\nu \beta} S_{+} k_{\beta}\right) e^{i k X}
$$

where $\eta=+1$ for Neumann directions and $\eta=-1$ for Dirichlet directions. Since the closed string vertex has four fermionic fields, the term with the minimal power of momenta is obtained by replacing the vertex operators by their fermionic terms only and furthermore by replacingthe fermionic fields by their zero modes. Schematically this would give rise to a term in the effective action of the form

$$
g^{2} \partial X \partial X \partial \partial \phi
$$

where $\phi$ denotes a massless closed string field and $X$ an open string field. At one loop this can contribute to the absorption of fixed scalars in the 1-brane 5-brane system and absorption of $l=2$ modes in the 3-brane system, since both these processes go as $g^{2}$ at the tree level. Note that at one loop there is no overall dilaton factor in front of the effective action as a result of which fixed scalars can interact with just two open string fields.

\footnotetext{
${ }^{6}$ I would like to thank C. Callan for a discussion on this point.
} 
When we have single branes (so that the gauge group is abelian), the planar diagram cancels the nonplanar diagram for the reason discussed above : the vertex operators do not involve any oscillators so that the insertion of a twist operator does not change the magnitude of the diagram. In so far as the effective string model is trustworthy at this level, this would mean that this does not alter the absorption/emission of fixed scalars. Similarly, this shows that the $l=2$ absorption by a single 3 -brane does not receive a $O\left(g^{2}\right)$ contribution from one loop.

This argument does not a priori apply to the case of $N$ 3-branes because of non-trivial Chan-Paton factors. One may wonder whether a nonzero contribution from such one loop diagrams may account for the difference between the brane and classical results in this case [19]. However this cannot be the case, since the absorption cross-section obtained after summing over the open string states would go as $g^{4} \alpha^{6} \omega^{7}$ as opposed to the tree level result $g^{4} \alpha^{\prime 8} \omega^{11}$. This is because the tree level diagram has more open string states which give higher powers of momentum from the phase space factors. This would make the one loop result dominate over the tree level result at low energies. While we do not have a good reason why this particular one loop contribution vanishes even for $N 3$-branes, we believe that the answer has got to do with the fact that to this order the 3-brane system behaves as a collection of $N^{2}$ abelian fields.

Finally we consider higher order (in string loop) corrections to terms in the 3-brane effective action which are responsible for the higher partial wave absorptions. The term for the $l$ th partial wave is given in (2.13) and involves $l$ derivatives on the closed string field and one derivative each on two fo the $(l+2)$ open string fields. We want to see whether there could be one-loop corrections to such terms.

Our analysis shows that any one-loop contribution must have at least four powers of the momentum, which may be distributed among the open and closed string fields, since there must be at least eight fermion zero modes. This immediately shows that there cannot be one-loop contributions to terms like (2.13) for $l=0,1$ since at tree level they do not have the necessary powers of momenta. Any loop correction to such processes would be therefore suppressed at low energies. On the other hand there could be in general nonzero contributions to $l \geq 2$ partial waves. Furthermore for such terms there is no cancellation between planar and nonplanar diagrams even for single branes since there are at least two open string fields with no momenta accompanying them. This could arise when the $\partial_{\sigma} X$ term in the corresponding vertex operators contribute to the one loop diagram as the necessary fermionic zero modes are already supplied by two open and one closed string 
field. This term necessarily involves oscillators which are indeed affected by the action of a twist operator. Detailed analysis of such diagrams are need : perhaps this is the reason why there is a discrepancy for the $l \geq 2$ absorption cross-sections.

\section{Remarks}

Some of the above results were derived for single or parallel branes and are directly applicable to the 3 -brane system. They suggest that for this system some of the absorption processes do not receive corrections from one loop. This could be the reason why the results agree with the semiclassical answers.

The five dimensional (or the four dimensional) black hole is described in terms of brane systems for which it is difficult to do microscopic calculations. However we believe that some of the results we have presented for single branes are relevant to this case as well since most of the results in fact follow from an effective string picture. Assuming that the system can be indeed modelled by such a long D-string, our results give evidence for the nonrenormalization of emission/absorption of S-wave minimally coupled scalars and fixed scalars. However the validity of this effective string picture at the loop level need to be examined.

Finally we would like to emphasize that even if one is able to prove that similar nonrenormalization theorems protect the lowest order tree level results, it does not immediately follow that the information loss problem is solved. What is really missing here is a clear understanding of the horizon physics in the problem. The curvature of the background comes from summing over open string loops. It remains to be seen whether there are non-perturbative effects which spoil correspondence of this perturbation theory with the physics of the horizon. In view of the spectacular agreement of the results it is tempting to speculate that there are none.

\section{Acknowledgements}

A very preliminary version of this work was presented at the "Workshop on String Theory, Field Theory and Quantum Gravity" held at Puri in December 1996. I thank the local organizers for organizing a wonderful conference. I would like to thank A. Dabholkar, A. Jevicki, J. Maldacena, A. Sen, S. Shenker and L. Susskind for valuable discussions and A. Tseytlin for a correspondence. I especially thank Samir Mathur for many discussions and I. Klebanov for enlightening discussions and comments on the manuscript. I would also like to thank the Physics Departments of Brown University and Rutgers University for hospitality during the completion of this work. 


\section{References}

[1] L. Susskind, hep-th/9309145; J. Russo and L. Susskind, Nucl. Phys. B 437 (1995) 611

[2] M. Duff and J. Rahmfeld, Phys. Lett. B345 (1995) 441, hep-th/9406105

[3] A. Sen, Nucl. Phys. B440 (1995) 421, hep-th/9411187 and Mod. Phys. Lett. A10 (1995) 2081.

[4] A. Strominger and C. Vafa, Phys. Lett. B379 (1996) 99, hep-th/9601029.

[5] J. Maldacena and A. Strominger, Phys. Rev. Lett. 77 (1996) 428, hep-th/9603060; C. Johnson, R. Khuri and R. Myers, Phys. Lett. B378 (1996) 78, hep-th/9603061

[6] J. Breckenridge, R. Myers, A. Peet and C. Vafa, hep-th/9602065; J. Breckenridge, D. Lowe, R. Myers, A. Peet, A. Strominger and C. Vafa, Phys. Lett. B381 (1996) 423

[7] C. Callan and J. Maldacena, Nucl. Phys. B 475 (1996) 645, hep-th/9602043.

[8] G. Horowitz and A. Strominger, Phys. Rev. Lett. 77 (1996) 2368, hep-th/9602051.

[9] A. Dhar, G. Mandal and S.R. Wadia, Phys. Lett. B388 (1996) 51, hep-th/9605234.

[10] S.R. Das and S.D. Mathur, Nucl. Phys. B478 (1996) 561, hep-th/9606185; Nucl. Phys. B482 (1996) 153, hep-th/9607149.

[11] S. Gubser and I. Klebanov, Nucl. Phys. B482 (1996) 173, hep-th/9608108.

[12] J. Maldacena and A. Strominger, Phys. Rev. D55 (1997) 861, hep-th/9609026.

[13] S. Gubser and I. Klebanov, Phys. Rev. Lett. 77 (1996) 4491, hep-th/9609076.

[14] S.P. de Alwis and K.Sato, hep-th/9611189

[15] S. Ferrara, R. Kallosh and A. Strominger, Phys. Rev. D52 (1995) 5412, hepth/9508072.; S. Ferrara and R. Kallosh, hep-th/9602136; hep-th/9603090; G. Gibbons, R. Kallosh and B. Kol, hep-th/9607108; B. Kol and A. Rajaraman, hep-th/9608126.

[16] C.Callan, S. Gubser, I. Klebanov and A. Tseytlin, hep-th/9610172.

[17] I. Klebanov and M. Krasnitz, hep-th/9612051

[18] I. Klebanov, hep-th/9702076

[19] S. Gubser, I. Klebanov and A. Tseytlin, hep-th/9703040

[20] S. Gubser, I. Klebanov and A. Peet, Phys. Rev. D54 (1996) 3915, hep-th/9602135; I.

Klebanov and A. Tseytlin, Nucl. Phys. B475 (1996) 165, hep-th/9604089

[21] G. Horowitz and J. Polchinski, hep-th/9612146.

[22] J. Maldacena, hep-th/9611125

[23] J. Maldacena and L. Susskind, Nucl. Phys B475 (1996) 679, hep-th/9604042.

[24] S.R. Das and S.D. Mathur, Phys. Lett. B375 (1996) 103, hep-th/9601152.

[25] S.D. Mathur, hep-th/9609053.

[26] J. Maldacena, Nucl. Phys. B477 (1996) 168, hep-th/9605016.

[27] A. Hashimoto and I. Klebanov, Phys. Lett B381 (1996) 437, hep-th/9604065.

[28] E. Witten, Nucl. Phys. B460 (1996) 541, hep-th/9511030.

[29] R. Leigh, Mod. Phys. Lett. A4 (1989) 2767; M. Li, Nucl. Phys. B460 (1996) 351; M. Douglas, hep-th/9512077; A.A. Tseytlin, Nucl. Phys. B469 (1996) 51. 
[30] S.R. Das, G. Gibbons and S.D. Mathur, Phys. Rev. Lett. 78 (1997) 417, hepth/9609052.

[31] I. Klebanov and S.D. Mathur, hep-th/9701187

[32] F. Dowker, D. Kastor and J. Traschen, hep-th/9702109.

[33] M. Green and M. Gutperle, Phys. Lett. B377 (1996) 28, hep-th/9602077; Nucl. Phys. B476 (1996) 9604091; K. Hamada, hep-th/9612234

[34] S. Gubser, A. Hashimoto, I. Klabanov and J. Maldacena, Nucl. Phys. B472 (1996) 231, hep-th/9601057.

[35] M. Garousi and R. Myers, Nucl. Phys. B475 (1996) 193, hep-th/9603194.

[36] M. Green, J. Schwarz and E. Witten, Superstring Theory, Vol. 2 (Cambridge University Press, 1986)

[37] A. Tseytlin, Phys. Lett. B367 (1996) 84, hep-th/9510173; Nucl. Phys. B467 (1996) 383, hep-th/9512081.

[38] T. Banks, W. Fischler, S. Shenker and L. Susskind, hep-th/9610043.

[39] C. Bachas, Phys. Lett. B374 (1996) 37,hep-th/9511043

[40] J. Maldacena and A. Strominger, hep-th/9702015.

[41] A. Abouelsaood, C.G. Callan, C.R. Nappi and S.A. Yost, Nucl. Phys. B280 [FS18] (1987) 599; C. Bachas and M. Porrati, Phys. Lett. B296 (1992) 77. 